This item was submitted to Loughborough's Research Repository by the author.

Items in Figshare are protected by copyright, with all rights reserved, unless otherwise indicated.

\title{
Adaptive driver modelling in ADAS to improve user acceptance: a study using naturalistic data
}

PLEASE CITE THE PUBLISHED VERSION

https://doi.org/10.1016/j.ssci.2018.08.023

PUBLISHER

Elsevier BV

VERSION

AM (Accepted Manuscript)

\section{PUBLISHER STATEMENT}

This paper was accepted for publication in the journal Safety Science and the definitive published version is available at https://doi.org/10.1016/j.ssci.2018.08.023.

\section{LICENCE}

CC BY-NC-ND 4.0

\section{REPOSITORY RECORD}

Fleming, James, Craig K Allison, Xingda Yan, Roberto Lot, and Neville A Stanton. 2018. "Adaptive Driver Modelling in ADAS to Improve User Acceptance: A Study Using Naturalistic Data”. Loughborough University. https://hdl.handle.net/2134/12623849.v1. 


\title{
Adaptive driver modelling in ADAS to improve user acceptance: a study using naturalistic data
}

\author{
James M. Fleming ${ }^{1}$, Craig K. Allison, Xingda Yan, Neville A. Stanton, Roberto Lot \\ School of Engineering, University of Southampton, UK
}

\begin{abstract}
Accurate understanding of driver behaviour is crucial for future Advanced Driver Assistance Systems (ADAS) and autonomous driving. For user acceptance it is important that ADAS respect individual driving styles and adapt accordingly. Using data collected during a naturalistic driving study carried out at the University of Southampton, we assess existing models of driver acceleration and speed choice during car following and when cornering. We observe that existing models of driver behaviour that specify a preferred inter-vehicle spacing in car-following situations appear to be too prescriptive, with a wide range of acceptable spacings visible in the naturalistic data. Bounds on lateral acceleration during cornering from the literature are visible in the data, but appear to be influenced by the minimum cornering radii specified in design codes for UK roadway geometry. This analysis of existing driver models is used to suggest a small set of parameters that are sufficient to characterise driver behaviour in car-following and curve driving, which may be estimated in real-time by an ADAS to adapt to changing driver behaviour. Finally, we discuss applications to adaptive ADAS with the objectives of improving road safety and promoting eco-driving, and suggest directions for future research.
\end{abstract}

Keywords: ADAS, Speed choice, Safe cornering, Car following, Driver modelling, Naturalistic driving,

\section{Introduction}

Advanced Driver Assistance Systems (ADAS), such as lane departure warning, curve warning and collision warning systems, are effective in reducing the incidence and severity of road accidents. Estimates of the reduction in the number of rear end collisions given by collision warning systems have been as high as $80 \%$ among distracted drivers, and the same system provides a safety benefit to attentive drivers by reducing the time required to release the accelerator before a potential crash (Lee et al., 2002). A lane departure warning was shown by Kozak et al. (2006) to decrease reaction time to a lane excursion by a factor of two among sleep-deprived drivers, with greater reductions when feedback was given in the form of vibration. ADAS have also been designed that promote fuel efficient driving (Staubach et al., 2014), motivated by studies showing that feedback is necessary to retain learned eco-driving behaviours (Froehlich et al., 2009).

In practice, the efficacy of ADAS is limited by user acceptance; even the most accurate warning system is useless if the driver disables it. Operator disablement of warning systems has been observed in situations as diverse as aeroplane cockpits (Patterson, 1982) and nuclear power plant control rooms (Seminara et al., 1977). After design choices such as the distinctiveness and loudness of alarms,

\footnotetext{
${ }^{1}$ Corresponding Author: J. M. Fleming, Mechanical Engineering, FEE, University of Southampton, UK. J.M.Fleming@soton.ac.uk
}

Sorkin (1988) implicates high false alarm rates as the most important factor limiting operator acceptance. For collision warning systems specifically, the probability of a crash given an alarm event is usually low due the low prior probability of collisions (Parasuraman et al., 1997), so that false alarms are quite common. User acceptance of curve warning systems for cars is also known to be problematic, as shown in operational tests (LeBlanc, 2006).

To reduce the number of false alarms generated by driver assistance systems while retaining a high sensitivity to potential accidents, good understanding of driver behaviour is vital. For example, an understanding of typical car-following behaviour is needed for effective collision warnings, so that false alarms are not generated during normal conditions. But following behaviour varies greatly between drivers, with Winsum and Heino (1996) showing time headways during car following ranging from $0.67 \mathrm{~s}$ to $1.52 \mathrm{~s}$, and minimum time-to-collision values during braking varying from $2.5 \mathrm{~s}$ to $5.1 \mathrm{~s}$. Likewise, to design curve warning systems that detect inattention and caution drivers to slow down, it is necessary to predict the speed at which an attentive driver would take a curve. In cornering, Reymond et al. (2001) demonstrates that typical lateral accelerations differ considerably from driver to driver with an observed range of $6.4 \mathrm{~m} / \mathrm{s}^{2}$ to $11.4 \mathrm{~m} / \mathrm{s}^{2}$. In addition to this variation between drivers, the behaviour of a single driver may also change depending on road conditions and driver fatigue (Brown, 1994).

A potential solution to this large variability in driv- 
ing behaviour is to design adaptive ADAS that modify their behaviour to fit the characteristics of a driver. This is made possible by the increasing number of sensors on modern vehicles. One such system has already been suggested by Wang et al. (2013), which recursively updates estimates of preferred time headway and driver sensitivity to deviations in headway and inverse time-to-collision. A key problem here is to identify parameters that are consistent at different times and hence may reliably be used to characterise the driver. Updating such a model in real-time requires that we describe the driver by a small number of measurable and physically meaningful parameters.

In the present paper, we review the existing literature on modelling of car-following and curve driving and critically evaluate these models using the results of a smallscale naturalistic driving study. The implications of this analysis for adaptive ADAS are discussed, considering what parameters of these models may be estimated by on-board sensors and the benefit in terms of user acceptance of collision and curve warning systems. Finally, naturalistic data analysis is used to recommend a set of parameters that may be used to characterise driver behaviour for adaptive ADAS. These can be used by the designers of driver assistance systems in order to improve user acceptance and therefore lead to better safety outcomes.

\section{ADAS for safety and eco-driving}

A total of 1,710 people lost their lives between July 2016 and June 2017 as a result of road accidents in the United Kingdom, and a further 174, 790 people were injured as a result of accidents (Department of Transport, UK Government, 2017). Since 2011, the number of people killed or injured on UK roads has fluctuated with no clear trend, and more work is needed to tackle road traffic accidents and minimise their transformative and potentially terminal impact. One approach to minimising this impact is via the use of ADAS (Marchau et al., 2005).

Estimates of the potential safety implications of ADAS are promising. Collision warning systems were shown in simulator studies to reduce the number of rear-end collisions by $80 \%$ (Lee et al., 2002), while on-road studies during the EuroFOT project have confirmed the positive effects of collision warning and adaptive cruise control systems on safety and fuel economy (Benmimoun et al., 2013). Similarly, a reduction of $59 \%$ in the number of fatal accidents (Carsten et al., 2000) is claimed to be viable with the extensive use of Intelligent Speed Adaptation systems. To achieve the goal of a positive impact on safety, such systems require high acceptance by their end users (Lindgren and Chen, 2006). This is especially true for warning systems where false alarm rates have been implicated in limiting operator acceptance (Sorkin, 1988). Within ADAS specifically, the need to explore the potential acceptance of system before implementation is necessary in order to achieve a positive safety impact (Biassoni et al., 2016).
The concept of an ADAS can be extended to not only support safety, but also encourage greater fuel efficiency and more environmentally friendly driving. In addition to the impact of vehicular drive trains (Chan, 2007) and mechanical systems (Vining, 2009) on fuel usage, the way a vehicle is driven can significantly influence fuel use and emissions. Research has estimated that $5-10 \%$ of fuel can be saved if drivers pursued a more fuel efficient, economical and environmentally friendly driving style referred to as eco-driving (Barkenbus, 2010), which is characterised by behaviours such as modest acceleration, early gear changes, minimising unnecessary braking, and driving below the speed limit. Recent analysis of naturalistic driving carried out as part of the UDRIVE project has indicated that braking, gear shifting and velocity choice on motorways each have effects on fuel consumption of $10 \%$ or more for conventional vehicles (Heijne et al., 2017).

Despite the advantages offered by a reduction in fuel usage and emissions, previous research has found that individuals typically struggle to maintain eco-driving behaviours long term, and rather are reliant on feedback to regulate their behaviour (Lauper et al., 2015). It has also been suggested that more individuals would adopt eco-driving if they understood the impact of their current actions (Abrahamse et al., 2005). Specifically examining transportation, (Froehlich et al., 2009) and (Meschtscherjakov et al., 2009) argue that providing feedback is a costeffective way to encourage and reinforce eco-driving practices. Based on this, it is clear that ADAS has the potential to facilitate both safer and more fuel-efficient driving. Such a system has already been evaluated in a driving simulator in Staubach et al. (2014), where a 15\% fuel saving was demonstrated by encouraging drivers to coast down before intersections.

\section{Models of driver behaviour}

\subsection{Car-following behaviour}

Modelling drivers' behaviour when following other vehicles has been the subject of active research since at least the mid-1940s (Herrey and Herrey, 1945). Much early research was motivated by applications to traffic management, and was successful in explaining emergent properties of traffic flow from the assumption that each driver behaves according to some simple rule. For example, Pipes (1953) considers the hypothesis that a driver adjusts their speed to maintain a 'legal distance' to a leader vehicle given by

$$
s=s_{0}+T_{0} v
$$

where $v$ is the vehicle speed. When applied to a line of vehicles this implies that each driver accelerates according to the relative velocity of the preceding vehicle, which is shown to cause velocity changes in the line of traffic that propagate as a wave. These waves are often observed in real-world traffic flow, for instance at intersections when a traffic signal turns green. 
Experimental evidence for a similar model of acceleration was given by Chandler et al. (1958), who proposed that the driver accelerates in proportion to the relative velocity of the vehicle ahead but with some finite reaction time, such that vehicle acceleration is given by the expression

$$
a\left(t+T_{r}\right)=\lambda\left[v_{l}(t)-v(t)\right]
$$

where $v$ and $a$ denote velocity and acceleration, $v_{l}$ denotes the velocity of the preceding vehicle, $\lambda$ is a parameter representing the sensitivity of the driver to velocity differences, and $T_{r}$ is the reaction time. An interesting feature of this model is that it can show instability for sufficiently large values of $\lambda$ or $T_{r}$. This instability may be the basis of further emergent properties of traffic flow such as jams or collisions (Chandler et al., 1958). A great number of subsequent works have suggested refinements and additions to these car-following models, as summarised in the review paper by Brackstone and McDonald (1999). Similarly to (Chandler et al., 1958), these models consist of an equation for vehicle acceleration expressing some dependence on velocity, lead vehicle velocity, and possibly the inter-vehicle spacing.

For the present paper our interest in these models is not that they may be used to simulate traffic flow (Yang and Koutsopoulos, 1996), but rather that they characterise driver behaviour by a small number of parameters that are measurable in real-time as part of an adaptive ADAS. In particular, this limits the usefulness of models that contain parameters that must be fit to data by using optimisation techniques (Kesting and Treiber, 2008) rather than having a simple physical meaning. The Intelligent Driver Model (IDM), proposed by Treiber et al. (2000), is useful in this regard as all parameters correspond to quantities that are readily estimated from velocity and range data. These model parameters are given in Table 1 along with their physical interpretations. Notably, this model assumes a spacing identical to that given by (1) when moving at a steady speed behind another vehicle.

\begin{tabular}{|l|l|}
\hline Parameter & Physical Interpretation \\
\hline$s_{0}$ & Min. distance to leader when stationary \\
\hline$T_{0}$ & Min. time headway to leader \\
\hline$a_{\max }$ & Max. desired longitudinal acceleration \\
\hline$b_{\max }$ & Max. desired longitudinal deceleration \\
\hline$v_{0}$ & Desired velocity \\
\hline
\end{tabular}

Table 1: Parameters of the IDM

Inter-vehicle spacings and car-following behaviour may also be studied in isolation, without considering the resulting traffic behaviour. Experiments performed on a test track by Winsum and Heino (1996) suggest that each driver has a preferred time headway that they maintain in car following situations. Similarly in (Brackstone et al., 2002 ) it is shown that in motorway driving, human drivers allow a following distance that increases linearly with speed, corresponding to an approximately constant time separation. Further work by the same authors (Brackstone et al., 2009) considers other factors that may affect following distances and concludes that they are unaffected by the level of traffic flow or the road geometry, but they are affected by the type of vehicle that is being followed, and may vary with time for a given driver.

From studies carried out in an early driving simulator, Todosiev (1963) noted that the acceleration and relative velocity of a driver following another vehicle shows a 'limit cycle' with maximum and minimum acceptable following distances. These cycles, visible as spirals when plotting vehicle spacing against relative velocity, are also observed in real-world driving (Brackstone et al., 2002). A perceptual basis for this phenomenon has been suggested, with the limit cycle behaviour due to physiological thresholds on detection of relative velocity (Todosiev, 1963). Later studies have developed this into a complete framework for describing car-following (Leutzbach and Wiedemann, 1986). Perhaps the most widely-cited work on driver perception in car following is (Lee, 1976), which advocates the use of 'time-to-collision' as a predictive variable for the onset and control of braking, based on a simplified analysis of the human visual system.

Some criticism has been levelled at the existing carfollowing models by Boer (1999), who argued that drivers perform many tasks simultaneously and as a result are typically satisfied with a range of conditions rather than having specific preferred vehicle spacings or speeds. This idea of 'satisficing' rather than 'optimising' originates in (Simon, 1955), which suggests that humans do not attempt to make optimal decisions, and instead classify outcomes as satisfactory or unsatisfactory and act accordingly.

\subsection{Cornering behaviour}

In the literature, steering has typically been considered as a control task independent of speed control, where the driver makes steering adjustments continuously in response to deviations in road position (McRuer et al., 1977). Similarly to driver response during car-following, experiments using driving simulators have shown limit cycles in the steering control of drivers when on a straight road that is consistent with the driver applying feedback subject to perceptual limits (Baxter and Harrison, 1979). This feedback is not the only aspect of steering control, as Godthelp (1985) demonstrated a precognitive aspect to steering by occluding the view of drivers during lane-change manoeuvres. These models have been refined through the application of control theory (Hess and Modjtahedzadeh, 1990) and modern developments have included consideration of the driver's neuromuscular dynamics (Pick and Cole, 2003), but they do not consider the effect of steering on the driver's speed choice, which is important to reduce false alarms in curve warning systems and to encourage coasting before curves in eco-driving assistance systems.

To model cornering speeds, Godthelp (1986) considered the role of the human visual system in cornering and 
showed that a quantity called 'time-to-lane crossing' is kept above a threshold by drivers negotiating a curve. This model predicts that drivers will choose lower speeds on tighter curves and narrower roads. This was further elaborated in (Reymond et al., 2001), which demonstrated that a minimum time-to-lane crossing is equivalent to an upper limit to the lateral acceleration of the vehicle while cornering. In experiments carried out on a test track, Reymond et al. (2001) further showed that drivers' lateral acceleration, denoted $\gamma$, has an upper limit that decreases when travelling at higher speeds according to the expression

$$
\gamma \leq \Gamma_{\max }-\Delta C_{\max } v^{2}
$$

where $\Gamma_{\max }$ is a driver parameter representing the limit on lateral acceleration tolerated by the driver at low speeds, while $\Delta C_{\max }$ determines the decrease of this limit with speed. As the lateral acceleration experienced while driving around a corner of curvature $\kappa$ is $\gamma=\kappa v^{2}$, the parameter $\Delta C_{\max }$ can be interpreted as a margin of error allowed by the driver when visually estimating the curvature of an upcoming corner. By substituting $\gamma=\kappa v^{2}$ into (3), this bound on lateral acceleration implies an upper bound on speed for a given corner given by:

$$
v \leq \sqrt{\frac{\Gamma_{\max }}{\kappa+\Delta C_{\max }}}
$$

It is interesting to note that this decrease of maximum lateral acceleration with speed cannot be explained from technical considerations of vehicle grip and handling, as the lateral acceleration at which the vehicle loses grip and skids is not dependent on speed (Gillespie, 1997).

Other models have been suggested in the literature that consider the curvature-speed relation directly. For example, Levison et al. (2007) provides an empirically-derived relationship predicting a limit on driver speed in terms of road curvature as $v \leq \alpha \kappa^{-1 / 4}$, where $\alpha$ is a driver parameter. This is equivalent to a speed-dependent lateral acceleration bound of

$$
\gamma \leq \frac{\alpha^{3}}{v^{2}}
$$

which allows for direct comparison with the model of (Reymond et al., 2001). Drawing from observed relationships between velocity and path curvature in human arm movements (Viviani and Schneider, 1991), and subsequent links to optimal control theory in the motor control literature (Viviani and Flash, 1995), Bosetti et al. (2015) suggests a 'two-thirds law' relationship between the maximum speed in curves and road curvature given by $v \leq \beta \kappa^{-1 / 3}$ which is equivalent to a lateral acceleration bound

$$
\gamma \leq \frac{\beta^{3}}{v}
$$

with $\beta$ the parameter characterising the driver. It has also been suggested that road width may affect driver speed choice in curves (DeFazio et al., 1992), although no account of curvature was taken in that study.

It is notable that much of the existing research into driver behaviour models, especially for following distances and cornering speeds, has been carried out on test tracks and in simulators. There is a gap in the literature in that comparatively little work has been done to validate these models in naturalistic conditions. The present study hopes to address this by starting to fill this gap.

\section{Methods}

\subsection{Hardware}

Naturalistic driving data was collected as part of a small-scale study, carried out at the university of Southampton in the UK, in which data was collected using a nonintrusive, portable, Automobile Data Acquisition Module (ADAM) (Yan et al., 2017) that attaches to the bottom corner of a car windscreen on the passenger side using a suction cup. The device then gathers naturalistic driving data, specifically time series data of position and velocity (via GPS), acceleration and angular rotation (via integrated accelerometers) as well as inter-vehicle spacing using a pair of stereo cameras. ADAM was designed to collect data as part of the G-ACTIVE research project currently being carried out at the University of Southampton and Imperial College London (http://www . g-active.uk), and has the advantage that it may easily be installed and removed by the participants in the study, without any modification to the vehicle. Because this process can be carried out daily by the participants themselves, it reduces the potential for demand characteristics whereby participants modify their behaviour in order to perform in a way pleasing to the researcher.

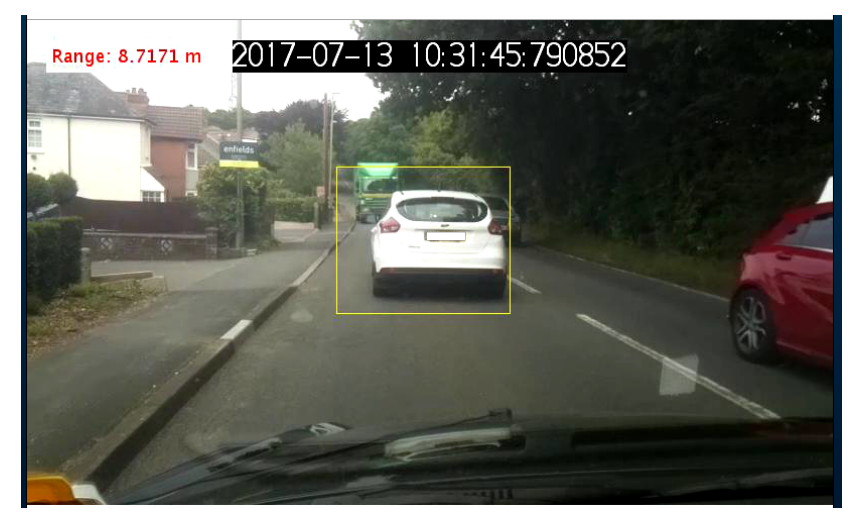

Figure 1: Example of video data from ADAM

The stereo video captured from ADAM may be postprocessed in order to provide the range to vehicles in front of the unit. A typical frame from the captured video, along with a headway value calculated from the stereo video cameras, can be seen in Figure 1. 


\subsection{Naturalistic Data Collection}

Data was collected from a total of 6 participants, denoted F1-F6, who regularly drove through urban areas with heavy traffic. These participants took the device for several days each and recorded their driving by installing ADAM in their own vehicles. They were not instructed to follow any particular route, instead following their usual journeys to gather data. After discarding data recorded at night time due to difficulties in using ADAM to estimate range in darkness, 7 hours of time-series data remained to assess car following behaviour.

To assess cornering behaviour, naturalistic data was collected from a further 3 participants, denoted C1-C3. Participant $\mathrm{C} 1$ had a daily commute through a rural area and contributed a total of 10 hours of data over 17 individual drives, most of which was in rural conditions where speed was not limited by traffic. Participants C2 and C3 contributed approximately an hour of data each using ADAM, again in rural conditions. Time-series data of GPS position from these participants was processed to provide an estimate of road curvature, and the resulting speed and acceleration data was filtered to remove noise. All participants (F1-6, C1-3) were frequent drivers aged between 25-40 with at least 3 years of experience, driving vehicles with engine sizes between 1.2-2.0 litres.

\section{Results}

\subsection{Car-following}

Initial analysis of the data revealed the limit cycles reported by Todosiev (1963) when the data is plotted in the 'phase space' of vehicle spacing versus relative velocity, which is defined such that positive values correspond to the participant's car travelling faster than the leading vehicle. A typical such spiral is shown in Figure 2 for a slow-moving car-following situation (mean velocity $1.5 \mathrm{~m} / \mathrm{s}$ ), with 60 seconds of data shown. The longitudinal accelerations of the drivers studied ranged between $-4.5 \mathrm{~m} / \mathrm{s}^{2}$ and $4 \mathrm{~m} / \mathrm{s}^{2}$, with no emergency braking events observed.

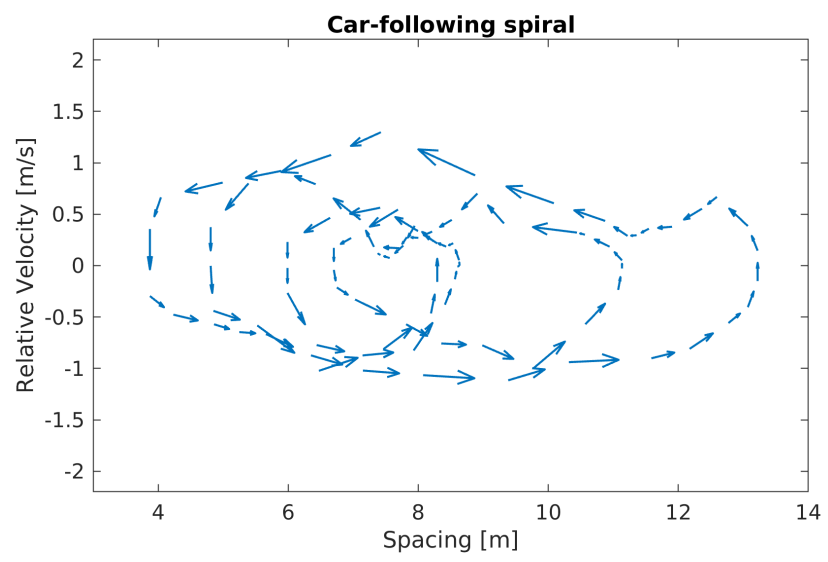

Figure 2: A typical car-following spiral
We investigated the relationship between velocity and vehicle spacing by filtering and down-sampling the collected time-series data to a sample rate of 1 s and retaining only those time instants where the absolute value of acceleration of both the participant's vehicle and the preceding vehicle was less than $0.5 \mathrm{~m} / \mathrm{s}^{2}$ (that is, both vehicles were travelling at approximately constant speed). The retained values of observed spacing are shown in Figure 3 for participant F1. There was a large variability in observed vehicle spacings for all participants, although a general trend of increasing spacing with increasing speed was observed. Figure 3 also shows the line of best fit obtained by linear regression, corresponding to the relationship (1), which gave $s_{0}=9.4 \mathrm{~m}$ and $T_{0}=0.90 \mathrm{~s}$ in this case. The wide variability in observed spacings and hence poor predictive value of this relationship is evident from the figure, with $R^{2}=0.51$.

For all participants, we also observed that the lower bound of the observed spacings increased with velocity and was well-approximated by a linear relationship as in (1). An estimate of this lower bound on observed spacing is shown in Figure 3 for participant F1, and the estimated values of $s_{\min }$ and $T_{\min }$ for the other participants are given in Table 2. The lack of points between $15-25 \mathrm{~m} / \mathrm{s}$ in the figure is due to steady following at these velocities rarely being observed in the naturalistic data. This is likely because the participants mostly drove on roads with speed limits of 30, 40 and 70 miles/hour.

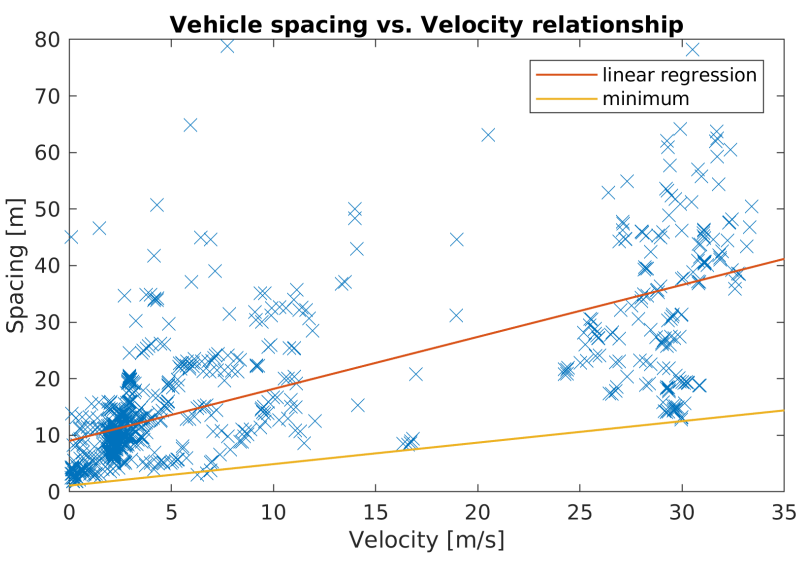

Figure 3: Observed vehicle spacings for F1

To investigate the Time-To-Collision (TTC) in carfollowing, we considered the relationship between spacing and relative velocity. The naturalistic time-series was again filtered and down-sampled to a rate of $1 \mathrm{~s}$, and all time instants when the participant car had a velocity of zero were removed. The result of this procedure is shown in Figure 4 for participant F1. From the figure, it is immediately apparent that there is a lower and upper bound to the observed relative velocities that appears to be wellapproximated by a linear relationship. Noting that the inverse of TTC corresponds to the slope of a line on this graph, the upper bound is consistent with the driver main- 
taining a TTC of greater than $2.4 \mathrm{~s}$.

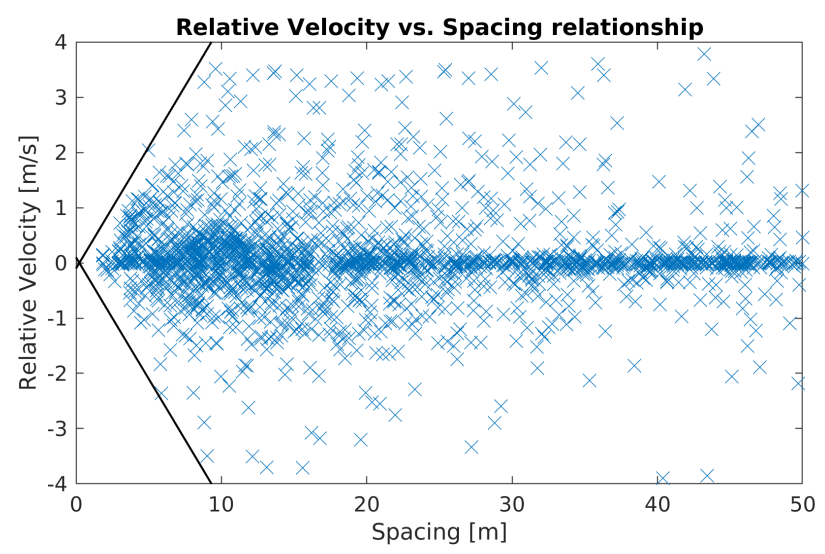

Figure 4: Observed relative velocities for $\mathrm{F} 1$

Minimum observed TTC values for the other study participants are shown in Table 2. We note also that the lower bound to observed TTC as shown in Figure 4 for participant F1, and the approximately linear lower bound on observed spacing shown in Figure 3, is observed in the data collected from the other study participants.

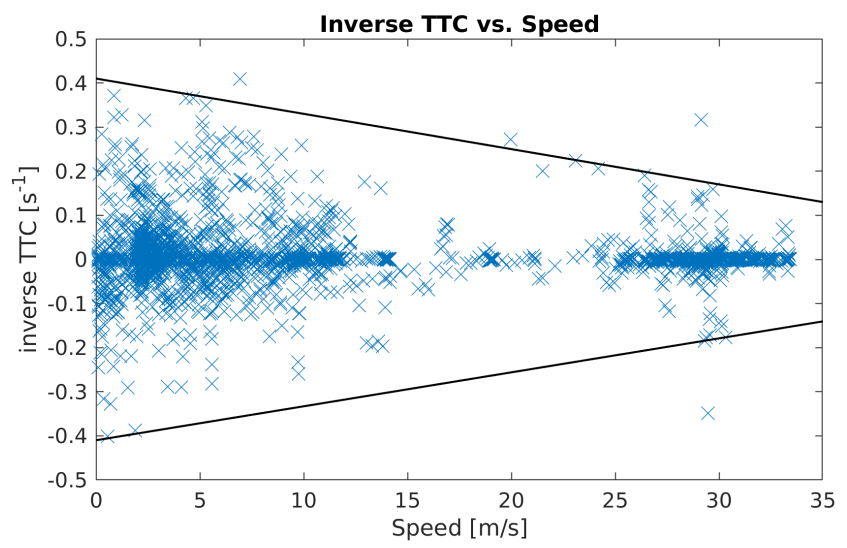

Figure 5: Observed inverse TTC for F1

Finally, we investigated the possible dependence of TTC on speed by computing the inverse TTC, as shown in Figure 5. This figure shows the inverse TTC and speed observed for F1 at different time instants. Generally, the maximum value of inverse TTC appears to decrease with speed (corresponding to a minimum TTC that increases with speed). There also appears to be a lower bound on the inverse TTC, increasing with speed. From inspection of the figure, it appears that there is some symmetry between the upper and lower bounds, and this is was also observed for the other participants.

\subsection{Cornering}

The time-series data from participants C1-C3 were analysed in order to obtain relationships involving driver speed choice when cornering. All of the local maxima of

\begin{tabular}{|l|l|l|l|}
\hline Participant & $\mathbf{T T C}_{\min }[\mathbf{s}]$ & $s_{\min }[\mathbf{m}]$ & $T_{\min }[\mathbf{s}]$ \\
\hline F1 & 2.40 & 1.34 & 0.38 \\
\hline F2 & 3.38 & 1.92 & 0.48 \\
\hline F3 & 3.13 & 1.45 & 0.45 \\
\hline F4 & 1.90 & 0.49 & 0.30 \\
\hline F5 & 3.21 & 1.44 & 0.64 \\
\hline F6 & 2.52 & 1.80 & 0.34 \\
\hline Mean & 2.76 & 1.41 & 0.43 \\
\hline S. d. & \pm 0.57 & \pm 0.50 & \pm 0.12 \\
\hline
\end{tabular}

Table 2: Observed minimum car-following parameters

curvature in the time series data were found, and for each curvature value the corresponding observed velocity was extracted. This procedure give a total of 7384 cornering events for C1, 657 for C2 and 730 for C3. These 'corners' included junctions and instances in which the speed of the vehicle was limited by traffic flow, the effect of which is to reduce the vehicle velocity to less than that attributable to cornering alone. The lateral acceleration was then estimated from the formula $\gamma=\kappa v^{2}$, which is the lateral acceleration when following a curved path, and the peak value of lateral acceleration in each corner extracted from the time-series. This provides a large set of observed lateral accelerations against velocity as shown in Figure 6.

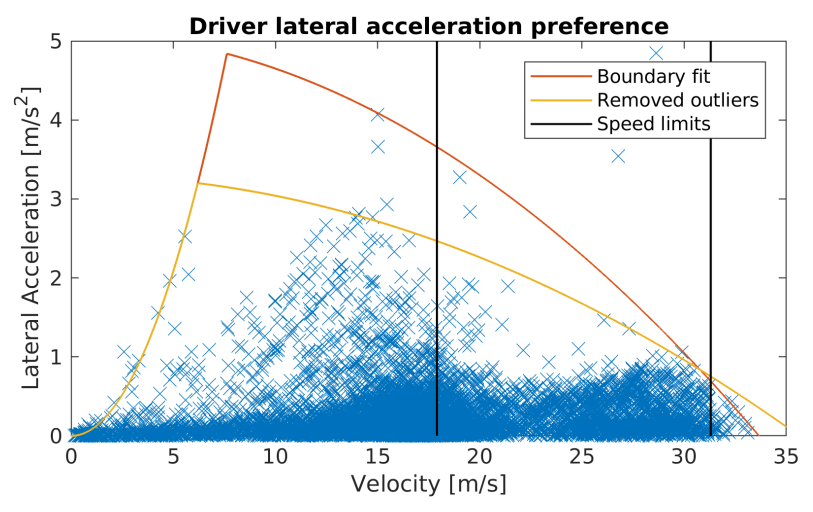

Figure 6: Observed lateral accelerations in cornering data

Following the procedure in Reymond et al. (2001), a 'high-velocity' upper bound curve following the relationship (3), and a 'low-velocity' curve from $\gamma=\kappa v^{2}$, were fitted to this data with some of the observed points classified as outliers and ignored. The low-velocity part of the bound is obtained assuming a minimum turning radius for the vehicle of $10 \mathrm{~m}$. Depending on the number of points designated as outliers, two possible curves are obtained with values of $\Gamma_{\max }=5.1$ and $\Delta C_{\max }=4.5$ or $\Gamma_{\max }=3.3$ and $\Delta C_{\max }=2.6$. It is evident that more corners have been observed with velocities of between $15-20 \mathrm{~m} / \mathrm{s}$ and also $25-30 \mathrm{~m} / \mathrm{s}$ than at other velocities. This uneven distribution of velocities is likely due to speed limits on the rural route that was most often driven by the participant. In particular, speed limits of 40 and 70 miles per hour were the most common, and we have indicated these val- 
ues with vertical lines in Figure 6. The high-speed part of this plot is shown in greater detail in Figure 7. Qualitatively, it appears from the latter figure that the red curve with values of $\Gamma_{\max }=5.1$ and $\Delta C_{\max }=4.5$ fits the data better at high speed, but this may be due to the relatively small number of corners observed above the speed limit of $70 \mathrm{mph}$ (corresponding to $31.3 \mathrm{~m} / \mathrm{s}$ ).

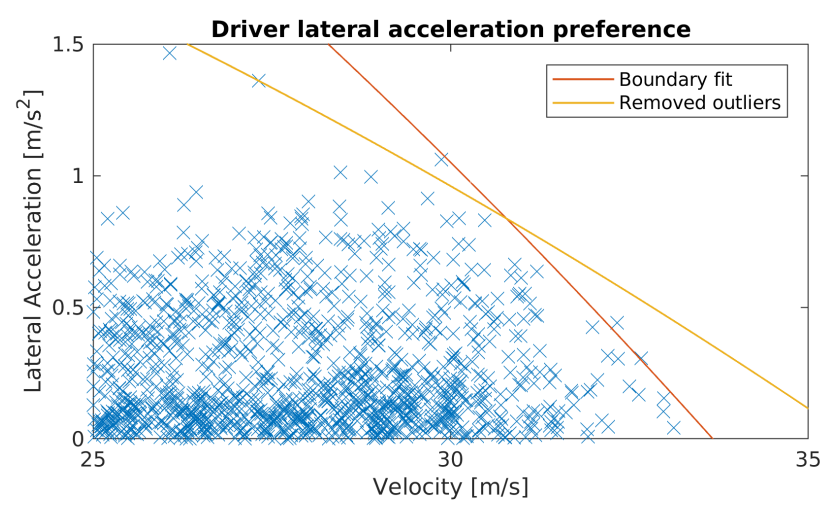

Figure 7: Detail of high-speed data

These lateral acceleration limits imply an upper bound to velocity that depends on curvature as shown in Figure 8 , which shows the velocities observed at the point of maximum curvature in each corner. It is notable that the outliers appear much closer to the rest of the data when plotting the velocity limit instead of the lateral acceleration. Also shown is a vertical line denoting the maximum recommended curvature for a $60 \mathrm{mph}$ road according to the UK design guidelines for road geometry (Highways Agency, UK Government, 2002). We conjecture that this causes the rapid apparent drop off in observed velocities visible in the figure near a curvature of $1.4 \mathrm{rad} / \mathrm{km}$, as curvatures of greater than this are only likely on roads with a speed limit of $50 \mathrm{mph}$ or less due to the design codes (Highways Agency, UK Government, 2002). This may also explain the low values of peak lateral acceleration observed in Figure 6 between the speeds of $20-25 \mathrm{~m} / \mathrm{s}$.

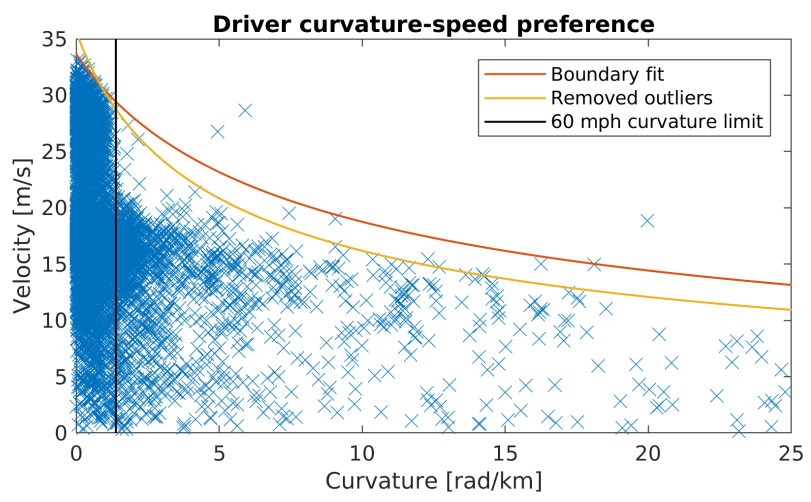

Figure 8: Observed velocities for cornering data

Other models of driver speed choice in curves were also evaluated against the gathered data. In particular, we as- sessed the 'two-thirds law' of Bosetti et al. (2015) and the power law relationship of Levison et al. (2007), both of which were suggested as models to predict driver speed in curves. Fits of these two relationships as upper bounds to the observed data are shown in Figure 9. Levison's relationship (Levison et al., 2007) appears to overestimate lateral acceleration for the corners with speeds of around $15 \mathrm{~m} / \mathrm{s}$ observed in the study. Conversely, the two-thirds law (Bosetti et al., 2015) appears to overestimate the allowable lateral acceleration at high speeds, such as for the numerous $30 \mathrm{~m} / \mathrm{s}$ corners seen in the data. Both of these models appear to allow very large lateral accelerations at speeds of around $10 \mathrm{~m} / \mathrm{s}$, such that they appear unlikely to provide a reasonable description of driver cornering speeds for large curvatures.

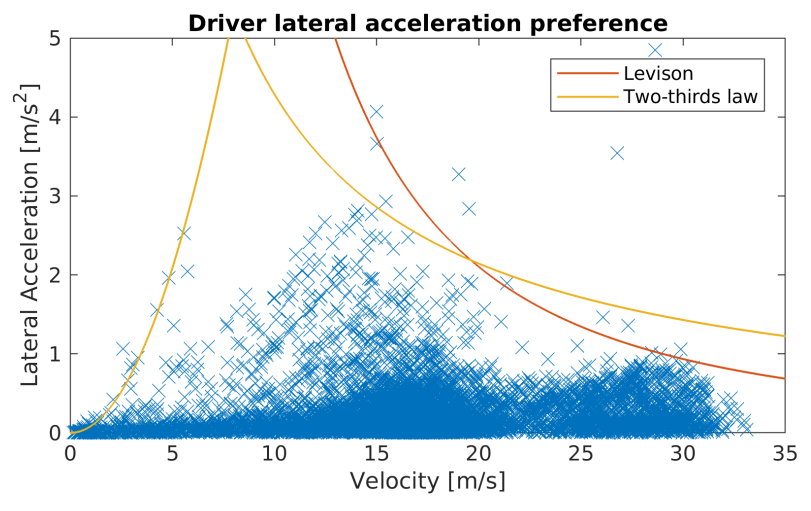

Figure 9: Alternative lateral acceleration models

The boundary fitting procedure was repeated for participants $\mathrm{C} 2$ and $\mathrm{C} 3$ to find the corresponding lateral acceleration bounds according to (3), and the resulting parameters are shown in Table 3 . It is notable that the values of the parameter $\Gamma_{\max }$ appear to be lower across our three participants C1-C3 than those given by Reymond et al. (2001), where the quoted mean was 7.64. A one-sample t-test for the null hypothesis $\mu=7.64$ yields $t(2)=-4.93$, $p<0.05$, suggesting significance, and the corresponding $95 \%$ confidence interval for the mean is [3.07, 7.27].

\begin{tabular}{|l|l|l|}
\hline Participant & $\Gamma_{\max }\left[\mathbf{m} / \mathbf{s}^{2}\right]$ & $\Delta C_{\max }[\mathbf{r a d} / \mathbf{k m}]$ \\
\hline C1 & 5.13 & 4.53 \\
\hline C2 & 5.34 & 4.95 \\
\hline C3 & 6.03 & 3.99 \\
\hline Mean & 5.98 & 4.49 \\
\hline S. d. & \pm 0.58 & \pm 0.49 \\
\hline
\end{tabular}

Table 3: Observed cornering parameters

\section{Discussion}

\subsection{Comparison of models with naturalistic data}

Preferred vehicle spacings were observed to vary widely, even when considering the same driver at different times. 


\begin{tabular}{|l|l|l|}
\hline Parameter & Typical Value & Physical Interpretation \\
\hline iTTC $_{\min }$ & $-0.2 \mathrm{~s}^{-1}$ & Minimum inverse time-to-collision to leading vehicle \\
\hline iTTC $_{\max }$ & $0.2 \mathrm{~s}^{-1}$ & Maximum inverse time-to-collision to leading vehicle \\
\hline$s_{\min }$ & $2 \mathrm{~m}$ & Minimum distance to leading vehicle when stationary \\
\hline$T_{\min }$ & $0.7 \mathrm{~s}$ & Minimum time headway to leading vehicle \\
\hline$a_{\max }$ & $4 \mathrm{~m} / \mathrm{s}^{2}$ & Maximum desired longitudinal acceleration \\
\hline$b_{\max }$ & $5 \mathrm{~m} / \mathrm{s}^{2}$ & Maximum desired longitudinal deceleration \\
\hline$\Gamma_{\max }$ & $6 \mathrm{~m} / \mathrm{s}^{2}$ & Maximum tolerable lateral acceleration \\
\hline$\Delta C_{\max }$ & $4 \mathrm{rad} / \mathrm{km}^{-1}$ & Driver curvature safety margin \\
\hline
\end{tabular}

Table 4: Recommended parameters for an ADAS driver model

This conflicts with the results of some previous studies such as (Winsum and Heino, 1996) which showed that drivers were consistent in their choice of headway, but this disparity may appear because that study was not done under naturalistic conditions. In particular, participants in (Winsum and Heino, 1996) were instructed to drive "as if they had to reach their destination as soon as possible, without overtaking other vehicles", which likely encouraged them to drive closer to the preceding vehicle. On the other hand, Brackstone et al. (2009) showed that for passive subjects who were unaware they were being studied, drivers were inconsistent in their choice of following distance. Brackstone et al. (2009) also observed a day-to-day variation in headway between the active participants in the study, supporting our conclusion that preferred following distances may vary widely for a particular driver.

A consequence is that many of the car-following models originating in the traffic literature, although successful at describing the bulk aspects of traffic flow, may be too prescriptive to accurately represent the behaviour of an individual driver. Many of these models, such as the Intelligent Driver Model (Treiber et al., 2000), include a preferred spacing for a given speed that will be approached in steady-state car following. Yet in our data we observed that individual drivers may remain at a steady speed at a wide variety of distances (Figure 3). We also observed the limit cycles described by Todosiev (1963) in the car following data (Figure 2), further suggesting a lack of a constant steady-state headway value. This has immediate implications for the design of adaptive collision warning systems, as it implies that it will be difficult to obtain a consistent estimate of time headway as attempted by Wang et al. (2013). We conjecture that driver behaviour is more accurately modelled if a minimum acceptable spacing is specified, which depends upon speed as in (1).

In contrast, when considering relative velocities there appears to be clearly defined minimum acceptable timeto-collision values (Figure 4), indicating that the onset of braking should be predictable according to the perceptual model of Lee (1976). Calculation of the inverse TTC reveals a dependence of the inverse TTC on speed, with an upper bound that decreases with speed, in agreement with the model in (Kiefer et al., 2005). We also observed a increasing lower bound on inverse TTC with speed that corresponds to the driver accelerating to catch up to the vehicle in front of them.

Of the cornering models considered, the lateral acceleration margin model of Reymond et al. (2001) appears to fit the collected naturalistic data better than Levison's model (Levison et al., 2007) and the two thirds law (Bosetti et al., 2015). Reymond's model also has the advantage of a wellexplained perceptual basis in that drivers allow a margin for error in their estimate of curvature when cornering. However, the are several quite subjective aspects to the model fitting, such as the elimination of outliers and hence the choice of points on the boundary when performing a regression analysis. For adaptive curve warning systems, we may infer that driver preferences are measurable if it is possible to reliably identify these outliers. A further complication is that design guidelines for road geometry affect the shape of the boundary and any adaptive ADAS should not fit to the resulting artificially low values of cornering speed that result from this (for instance, the region around 2-3 rad/km in Figure 8). These driver lateral acceleration preferences are also relevant for occupant comfort in autonomous driving, where user acceptance is likely to be low if the driver perceives the car to be going too fast or too slow in corners.

\subsection{Parameters characterising driver behaviour}

Generally, the most successful models in our analysis are those that prescribe bounds on quantities, such as the lateral acceleration bound of (Reymond et al., 2001) or the time-to-collision bound of (Lee, 1976), rather than those giving expected values, such as the preferred headway of Winsum and Heino (1996). This fits with the idea of a driver 'satisficing', that is ensuring satisfaction of constraints rather than optimising a performance criterion (Boer, 1999). Knowledge of these constraints is essential to understand typical driver behaviour, and hence for user acceptance of ADAS. A suggested set of parameters to be used to characterise a driver for adaptive ADAS is given in Table 4, and we now briefly justify these choices considering curve warning and collision warning systems.

The inverse time-to-collision (TTC) may be a more useful measure than time-to-collision, as in the data there appears to be an upper and lower bound to this quantity that 
may reduce in magnitude as vehicle velocity increases. Inverse TTC has already been suggested for use in forward collision warning systems, for example by Kiefer et al. (2005), and was also suggested as an input to an adaptive collision warning system (Wang et al., 2013). The observation that TTC appears to have a dependence on speed is consistent with the results in (Kiefer et al., 2005).

It is possible that the minimum observed spacing is consistent for each driver, as illustrated in Figure 3. However, the concept of a preferred spacing is in general not borne out by the naturalistic data, with linear regressions typically giving poor correlations of $R^{2} \approx 0.5$. This has interesting implications for forward collision warning systems specifically, as the desired inter-vehicle spacing is not predictable from the current velocity and if using low values of headway to trigger warnings, care should be taken in the design not to sound false alarms. Notably the adaptive ADAS of (Wang et al., 2013) uses this concept of a preferred spacing, despite the large variability we observed in this quantity during naturalistic driving. A much more consistent measure in our data is the minimum TTC (or equivalently, inverse TTC), which appears to have a welldefined acceptable range, and this quantity should be preferred when designing collision warning systems.

Naturalistic data on cornering speeds matches the model given in (Reymond et al., 2001) once allowance has been made for the limits of the road geometry, and this gives either an upper bound to the lateral acceleration or equivalently an upper bound to speed as a function of curvature that should be considered in the design of curve-warning systems. For our participants the observed values of the maximum lateral acceleration parameter $\Gamma_{\max }$ are significantly $(p<0.05)$ lower than those given in (Reymond et al., 2001), giving some evidence that the values in that paper may be too high for the naturalistic driving studied. It is possible that this represents a difference between naturalistic driving and driving on a test track as in (Reymond et al., 2001), although our sample size is also very small and it could represent differences in the participants used in the study. Given that the parameters defining this bound show considerable variation between drivers, a curve-warning system should adapt to observed values of lateral acceleration to minimise false alarms and improve operator acceptance. This seems especially important as operational tests of current curve warning systems have shown limited user acceptance (LeBlanc, 2006).

\subsection{Limitations}

The main limitation of the present work is the small number of participants used to gather the naturalistic data. As a result of this, the numerical values of the driving parameters considered in Tables 4 and 3 should not be considered representative of drivers as a whole. Rather, the main value lies in the investigation of the intra-driver variation of quantities such as following distance and cornering velocity, and identification of the models in the existing literature that give a good description of the behaviour of individual participants. We also note that all vehicle following data was collected in the daytime, and that different models may apply during hours of darkness.

The GPS unit of ADAM has a manufacturer-stated positional error of $\pm 0.9 \mathrm{~m}$ and a velocity error of $\pm 0.1 \mathrm{~m} / \mathrm{s}$. A 'worst-case' error analysis of the error when estimating road curvature based on these values gives $\pm 0.177 \mathrm{rad} / \mathrm{km}$ for a corner of curvature $10 \mathrm{rad} / \mathrm{km}$, although in practice the error is likely to be smaller as the curvature is estimated from several consecutive position, speed and heading measurements. Similarly, the lateral acceleration estimation error is $\pm 0.070 \mathrm{~m} / \mathrm{s}^{2}$ when travelling at $15 \mathrm{~m} / \mathrm{s}$ around a corner of curvature $10 \mathrm{rad} / \mathrm{km}$. The distance estimation algorithm of ADAM used to calculate intervehicle spacing has a range error of less than $0.1 \mathrm{~m}$ and a relative velocity error of less than $0.1 \mathrm{~m} / \mathrm{s}$ for distances of up to $40 \mathrm{~m}$, which were determined by the authors by comparison with data from a doppler radar installed on an instrumented vehicle.

\section{Conclusions}

Motivated by the high efficacy (Lee et al., 2002; Kozak et al., 2006) but low reported user acceptance (LeBlanc, 2006; Parasuraman et al., 1997) of some driver assistance systems, we have advocated the design of ADAS that adapt to the characteristics of a driver. In doing so, the number of false alarms can be decreased for warning systems, which are a major cause of user disablement (Sorkin, 1988). But to achieve this, accurate understanding of driver behaviour in naturalistic conditions is crucial.

Using naturalistic data collected as part of a study at the University of Southampton, we evaluated the usefulness of existing models in describing observed relationships in the data during car-following and cornering, with a focus on following distance, time-to-collision, cornering speed and lateral acceleration. In particular:

- There is a poor observed correlation between speed and inter-vehicle spacing. Speed appears to be a poor predictor for a driver's following distance, with drivers showing a large variation in their following distances at a given speed in the naturalistic data. This calls into question the use of models of this type in the design of collision-warning systems.

- Inverse time-to-collision (TTC) has a well-defined lower and upper bound for each driver that reduces in magnitude at higher speeds. This may be used to predict the onset of braking and acceleration in following situations, a finding that reinforces its use to identify critical situations for collision-warning.

- Speed while cornering is well-described by the lateral acceleration margin model of Reymond et al. (2001), which gives a better description of tolerable lateral accelerations than competing models and is 
a good candidate for use in curve warning and ecodriving assistance systems, although values of the model parameters appear lower in our data than in the original study.

Finally, we note that our analysis of the naturalistic data supports the notion that a driver satisfices rather than optimises when driving (Boer, 1999). For adaptive ADAS, we have therefore advocated a modelling approach using parameters that describe limits to quantities such as time headway, time-to-collision and lateral acceleration. This will allow future driver assistance systems to adapt to driver preferences, improving user acceptance and hence improving safety outcomes.

\section{Acknowledgements}

We gratefully acknowledge the support of the Engineering and Physical Sciences Research Council under grant number EP/N022262/1.

\section{References}

Abrahamse, W., Steg, L., Vlek, C., Rothengatter, T., 2005. A review of intervention studies aimed at household energy conservation. Journal of environmental psychology 25 (3), 273-291.

Barkenbus, J. N., 2010. Eco-driving: An overlooked climate change initiative. Energy Policy 38 (2), 762-769.

Baxter, J., Harrison, J. Y., 1979. A nonlinear model describing driver behavior on straight roads. Human Factors 21 (1), 87-97.

Benmimoun, M., Pütz, A., Zlocki, A., Eckstein, L., 2013. eurofot: Field operational test and impact assessment of advanced driver assistance systems: Final results. In: Proceedings of the FISITA 2012 World Automotive Congress. Springer, pp. 537-547.

Biassoni, F., Ruscio, D., Ciceri, R., 2016. Limitations and automation. the role of information about device-specific features in ADAS acceptability. Safety Science 85, 179-186.

Boer, E. R., 1999. Car following from the drivers perspective. Transportation Research Part F: Traffic Psychology and Behaviour 2 (4), 201-206.

Bosetti, P., Da Lio, M., Saroldi, A., 2015. On curve negotiation: From driver support to automation. IEEE Transactions on Intelligent Transportation Systems 16 (4), 2082-2093.

Brackstone, M., McDonald, M., 1999. Car-following: a historical review. Transportation Research Part F: Traffic Psychology and Behaviour 2 (4), 181-196.

Brackstone, M., Sultan, B., McDonald, M., 2002. Motorway driver behaviour: studies on car following. Transportation Research Part F: Traffic Psychology and Behaviour 5 (1), 31-46.

Brackstone, M., Waterson, B., McDonald, M., 2009. Determinants of following headway in congested traffic. Transportation Research Part F: Traffic Psychology and Behaviour 12 (2), 131-142.

Brown, I. D., 1994. Driver fatigue. Human factors 36 (2), 298-314.

Carsten, O., Fowkes, M., Tate, F., 2000. Implementing intelligent speed adaptation in the UK: Recommendations of the EVSC project. In: Proceedings of the 7th World Congress on Intelligent Transport Systems, ITS Congress Association, Brussels.

Chan, C. C., 2007. The state of the art of electric, hybrid, and fuel cell vehicles. Proceedings of the IEEE 95 (4), 704-718.

Chandler, R. E., Herman, R., Montroll, E. W., 1958. Traffic dynamics: studies in car following. Operations research 6 (2), 165-184.

DeFazio, K., Wittman, D., Drury, C., 1992. Effective vehicle width in self-paced tracking. Applied ergonomics 23 (6), 382-386.

Department of Transport, UK Government, 2017. Reported road casualties in Great Britain: quarterly provisional estimates year ending June 2017. https://www.gov.uk/government/ uploads/system/uploads/attachment_data/file/654962/ quarterly-estimates-april-to-june-2017.pdf, accessed: $11 / 12 / 2017$

Froehlich, J., Dillahunt, T., Klasnja, P., Mankoff, J., Consolvo, S., Harrison, B., Landay, J. A., 2009. Ubigreen: investigating a mobile tool for tracking and supporting green transportation habits. In: Proceedings of the SIGCHI Conference on Human Factors in Computing Systems. ACM, pp. 1043-1052.

Gillespie, T. D., 1997. Vehicle dynamics. Warren dale.

Godthelp, H., 1986. Vehicle control during curve driving. Human Factors 28 (2), 211-221.

Godthelp, J., 1985. Precognitive control: open-and closed-loop steering in a lane-change manoeuvre. Ergonomics 28 (10), 1419-1438.

Heijne, V., Ligterink, N., Stelwagen, U., 2017. Potential of ecodriving. UDRIVE Deliverable D45.1. EU FP7 Project UDRIVE Consortium. https://doi.org/10.26323/UDRIVE_D45.1.

Herrey, E. M., Herrey, H., 1945. Principles of physics applied to traffic movements and road conditions. American journal of physics 13 (1), 1-14.

Hess, R., Modjtahedzadeh, A., 1990. A control theoretic model of driver steering behavior. IEEE Control Systems Magazine 10 (5), 3-8.

Highways Agency, UK Government, 2002. Design manual for roads and bridges: Volume 6 road geometry. http://www.standardsforhighways.co.uk/ha/standards/dmrb/ vol6/section2/td4295.pdf, accessed: 11/12/2017.

Kesting, A., Treiber, M., 2008. Calibrating car-following models by using trajectory data: Methodological study. Transportation Research Record 2088 (1), 148-156.

Kiefer, R. J., LeBlanc, D. J., Flannagan, C. A., 2005. Developing an inverse time-to-collision crash alert timing approach based on drivers last-second braking and steering judgments. Accident Analysis \& Prevention 37 (2), 295-303.

Kozak, K., Pohl, J., Birk, W., Greenberg, J., Artz, B., Blommer, M., Cathey, L., Curry, R., 2006. Evaluation of lane departure warnings for drowsy drivers. In: Proceedings of the human factors and ergonomics society annual meeting. Vol. 50. Sage Publications Sage CA: Los Angeles, CA, pp. 2400-2404.

Lauper, E., Moser, S., Fischer, M., Matthies, E., Kaufmann-Hayoz, R., 2015. Psychological predictors of eco-driving: A longitudinal study. Transportation research part F: traffic psychology and behaviour 33, 27-37.

LeBlanc, D., 2006. Road departure crash warning system field operational test: methodology and results. volume 1: technical report.

Lee, D. N., 1976. A theory of visual control of braking based on information about time-to-collision. Perception 5 (4), 437-459.

Lee, J. D., McGehee, D. V., Brown, T. L., Reyes, M. L., 2002. Collision warning timing, driver distraction, and driver response to imminent rear-end collisions in a high-fidelity driving simulator. Human factors 44 (2), 314-334.

Leutzbach, W., Wiedemann, R., 1986. Development and applications of traffic simulation models at the karlsruhe institut für verkehrswesen. Traffic engineering \& control 27 (5), 270-278.

Levison, W. H., Campbell, J. L., Kludt, K., Bittner, A. C., Potts, I., Harwood, D. W., Hutton, J., Gilmore, D., Howe, J. G., Christos, J. P., et al., 2007. Development of a driver vehicle module for the interactive highway safety design model. Federal Highway Administration, Office of Research and Technology Services.

Lindgren, A., Chen, F., 2006. State of the art analysis: An overview of advanced driver assistance systems (ADAS) and possible human factors issues. Human factors and economics aspects on safety, 3850 .

Marchau, V., Van der Heijden, R., Molin, E., 2005. Desirability of advanced driver assistance from road safety perspective: the case of ISA. Safety Science 43 (1), 11-27.

McRuer, D. T., Allen, R. W., Weir, D. H., Klein, R. H., 1977. New results in driver steering control models. Human factors 19 (4), 381-397.

Meschtscherjakov, A., Wilfinger, D., Scherndl, T., Tscheligi, M., 2009. Acceptance of future persuasive in-car interfaces towards a more economic driving behaviour. In: Proceedings of the 1st 
International Conference on Automotive User Interfaces and Interactive Vehicular Applications. ACM, pp. 81-88.

Parasuraman, R., Hancock, P. A., Olofinboba, O., 1997. Alarm effectiveness in driver-centred collision-warning systems. Ergonomics 40 (3), 390-399.

Patterson, R. D., 1982. Guidelines for auditory warning systems on civil aircraft. Civil Aviation Authority.

Pick, A., Cole, D., 2003. Neuromuscular dynamics and the vehicle steering task. The Dynamics of Vehicles on Roads and on Tracks 41, 182-191.

Pipes, L. A., 1953. An operational analysis of traffic dynamics. Journal of applied physics 24 (3), 274-281.

Reymond, G., Kemeny, A., Droulez, J., Berthoz, A., 2001. Role of lateral acceleration in curve driving: Driver model and experiments on a real vehicle and a driving simulator. Human factors 43 (3), 483-495.

Seminara, J. L., Gonzalez, W., Parsons, S. O., 1977. Human factors review of nuclear power plant control room design. Electric power research institute.

Simon, H. A., 1955. A behavioral model of rational choice. The quarterly journal of economics 69 (1), 99-118.

Sorkin, R. D., 1988. Why are people turning off our alarms? The Journal of the Acoustical Society of America 84 (3), 1107-1108.

Staubach, M., Schebitz, N., Köster, F., Kuck, D., 2014. Evaluation of an eco-driving support system. Transportation research part F: traffic psychology and behaviour 27, 11-21.

Todosiev, E. P., 1963. The action point model of the driver-vehicle system. Ph.D. thesis, The Ohio State University.

Treiber, M., Hennecke, A., Helbing, D., 2000. Congested traffic states in empirical observations and microscopic simulations. Physical review E $62(2), 1805$.

Vining, C. B., 2009. An inconvenient truth about thermoelectrics. Nature materials 8 (2), 83-85.

Viviani, P., Flash, T., 1995. Minimum-jerk, two-thirds power law, and isochrony: converging approaches to movement planning. Journal of Experimental Psychology: Human Perception and Performance 21 (1), 32.

Viviani, P., Schneider, R., 1991. A developmental study of the relationship between geometry and kinematics in drawing movements. Journal of Experimental Psychology: Human Perception and Performance 17 (1), 198-218.

Wang, J., Zhang, L., Zhang, D., Li, K., 2013. An adaptive longitudinal driving assistance system based on driver characteristics. IEEE Transactions on Intelligent Transportation Systems 14 (1), $1-12$.

Winsum, W. v., Heino, A., 1996. Choice of time-headway in carfollowing and the role of time-to-collision information in braking. Ergonomics 39 (4), 579-592.

Yan, X., Fleming, J., Allison, C., Lot, R., 2017. Portable automobile data acquisition module (ADAM) for naturalistic driving study. In: Proceedings of the 15th European Automotive Congress.

Yang, Q., Koutsopoulos, H. N., 1996. A microscopic traffic simulator for evaluation of dynamic traffic management systems. Transportation Research Part C: Emerging Technologies 4 (3), 113-129. 